\title{
DETERMINATION OF SELF-OSCILLATIONS IN RELAY CONTROL SYSTEMS
}

\author{
ABDELOUAHAB ZAATRI ${ }^{1}$, RIDHA KELAIAIA ${ }^{2}$ \\ ${ }^{1}$ Independent Researcher, Ex-Dep. of Mechanical Engineering, Univ. of Brothers Mentouri, Constantine 1, Algeria, \\ ${ }^{2}$ LGMM Laboratory, Université 20 août 1955-Skikda, PB Nº 26 Route Elhadaik, Skikda 21000, Algeria \\ E-mail: azaatri@yahoo.com,r.kelaiaia@univ-skikda.dz
}

\begin{abstract}
Some dynamical linear elements including on-off elements such as electro-mechanical relays and electronic components used in switching mode for some feedback control systems can present special features such as the capability to autonomously generate stable self-oscillations. This paper concerns the comparison of two approaches enabling to determine the frequency of self-oscillations in these systems. We examine Tsypkin's approach which can provide analytical solutions to determining the frequency of existing selfoscillations. On the other hand, we examine the Describing Function (DF) approach which has been developed as an alternative to approximate these solutions.

We will compare these two approaches for nonlinear systems of first and second orders. We will examine particularly the possibilities and limits of each approach for calculating the self-oscillations. Simulation of these systems will be performed to visualizes their behaviors. An experimental feedback control system based on electronic circuits used in switching mode has been built as a setup enabling testing and envisioning some applications.
\end{abstract}

Keywords: On-Off control systems, relay feedback control, Tsypkin's method, Describing Function, Nonlinear modeling

\section{INTRODUCTION}

Nonlinearity is usually a serious issue in control engineering systems because there are no general methods to solve these types of problems. Non linearity is mostly inherent to the system to be controlled or to other elements that are involved in the structure of the feedback control systems. However, there are cases where the presence of some particular nonlinear elements may be intentionally used because they can provide a specific useful behavior for system control.

Historically, the most famous nonlinear elements that have initially and intentionally been used and then studied are relays [1, 2]. They have been firstly used as electromechanical devices for their on-off switching capability. Later on, they were followed by some electronic devices also used as switching devices such as transistors, thyristors, etc. Under certain conditions, on-off devices with and without hysteresis when included into feedback control systems may autonomously generate stable self-oscillations [3, 4]. Because of this propriety, they have gained a new interest since they have been successfully used as auto-tuners in control applications to tune PID controllers [5, 6]. In fact, they have also been used in various applications such as delta-sigma modulators for analog-digital converters [7], in robust relay-based controls, in optimal control, in nonlinear systems analysis and identification, and others [8,9]. From the viewpoint of practical applications, relays and other on-off switching devices were used at the beginning as electromechanical devices in power amplifiers [2, 3]. Later on, they have been used in many feedback control techniques such as flight controllers [10,11], missiles [12], marine [13], pulse with modulation generators, tracking systems, design of oscillators, etc. [14, 15].

The determination of self-oscillations is an important issue in the analysis and design of such feedback control systems. Some well-known approaches which have been developed in order to determine the self-oscillations are Tsypkin's approach [3], Phase Plane approach [16], and the DF approach [17]. Exact conditions for the establishment of limit cycle oscillations under relay feedback were developed by Hamel [4] and Tsypkin [3].

The present paper concerns the determination of the frequency of self-oscillations which are generated by a feedback system including an on-off element with hysteresis and a dynamical linear system. Mathematical expressions for determining the self-oscillations derived by means of Tsypkin's approach based on Laplace transform and Cauchy's theorem of residues are presented. These derived expressions enable to analytically determine, in case of no excitation, the self-oscillation frequency in relationship to the parameters of the on-off system and the linear system. On the other hand, we make use of the Describing Function approach to approximate this frequency. A comparison will be made between these two methods applied to systems of first and second orders. We will examine what are the possibilities and the limits of these two considered approaches concerning the determination of self-oscillations. We will exploit also the graphical results provided by numerical simulation and experimental results if possible.

\section{THE MODEL OF ON-OFF CONTROL SYSTEMS}

\subsection{The structure of the feedback control system}

A model of the feedback control system based on an onoff element followed by a linear system is schematically presented in Figure 1. It has been already described in [15]. It is constituted, in the direct chain, by a symmetrical nonlinear $+/$ - with a hysteresis of width noted $h$ and an 
extremal value +/- $E$. The involved variables are: the reference signal $f_{0}$ assumed null; the control signal $x(t)$, the controlled signal $u(t)$, the feedback signal $z(t)$, and the output signal $v(t)$ (Figure 1).

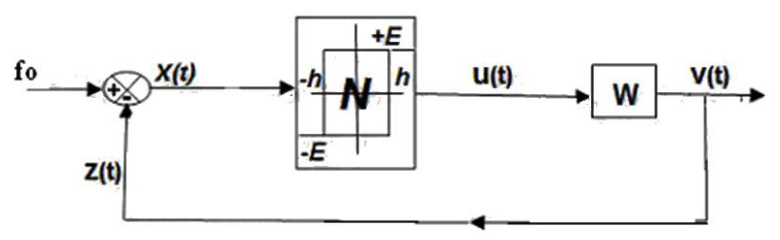

Figure 1. Scheme of the on-off linear control system

\subsection{The nonlinear behavior of the On-Off element " $N$ "}

For purpose of simplicity, we assume that the input voltage $x(t)$ has a triangular shape and can cross the threshold values $(+/-h)$ at specific moments. The output $u(t)$ will be a succession of pulses of constant amplitude of alternating sign $(+/-E)$ with variable duration and variable on-off intervals. The instants during which the jumps happen are called the switching instants. These instants are determined by the following conditions:

$$
\left\{\begin{array}{l}
\text { if } x(t)>+h \text { and } \frac{d x(t)}{d t}>0 \Rightarrow U(t)=-E \\
\text { if } x(t)<-h \text { and } \frac{d x(t)}{d t}<0 \Rightarrow U(t)=+E
\end{array}\right.
$$

An example of evolution of the output signal in correspondence with respect to the input signal is shown in Figure 2.

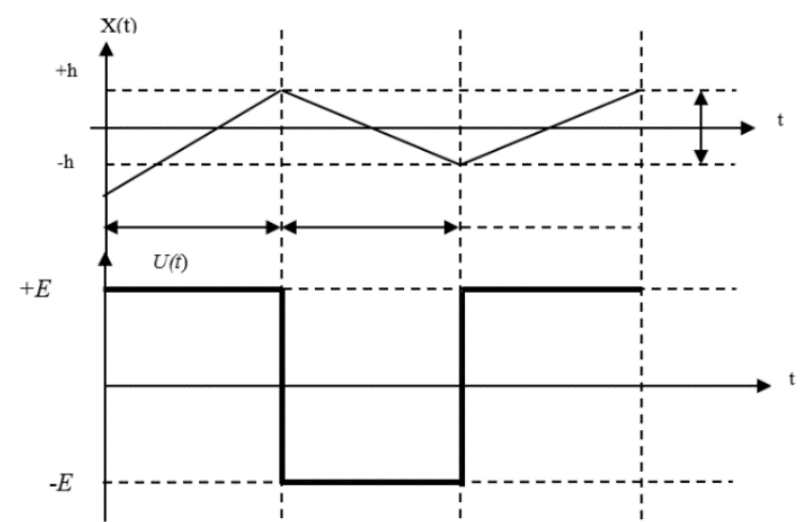

Figure 2. The input and output signals $X(t), U(t)$.

The determination of self-oscillations is a fundamental problem in the analysis of on-off systems. Among many techniques, we will examine in the following sections the Tsypkin's approach and the DF approach which intend to solve this problem $[17,18]$. In the general case, obtaining an analytical solution can be extremely difficult. When facing complexity to find solutions, some graphical techniques, simulation and tests on a home-made experimental system will be used to analyze this type of problems.

\section{TSYPKIN'S APPROACH}

Tsypkin's approach serves to analyze on-off feedback systems and enables to determine theoretically the self- oscillations by means of mathematical expressions. These expressions link the self-oscillation frequencies with respect to the characteristics of the nonlinear element: extreme magnitude $\mathrm{E}$ and hysteresis width $\mathrm{h}$; and of the linear element $\left(\tau=\frac{1}{s_{0}}, K\right)$. The presented technique of calculation of the self-oscillation is derived from Tsypkin's approach [3]. In case there is no excitation $\left(f_{0}\right)$; then:

$$
x(t)=v(t)=-z(t)
$$

Therefore, the signal $u(t)$ is a periodic function in the form of a square wave. By using Laplace transform, it can be expressed as:

$$
U(s)=\frac{E}{s} \operatorname{th}\left(\frac{T s}{4}\right)
$$

where $s$ is the Laplace variable, $t h($.$) is the hyperbolic$ tangent function and $T$ is the period of the self-oscillation we want to determine. The corresponding transfer function $W(s)$ of the linear system having the input $u(t)$ and the output $z(t)$ can be expressed as:

$$
W(s)=\frac{U(s)}{X(s)}
$$

By expressing, the relation (2) using Laplace Transform, we get:

$$
\begin{gathered}
X(s)=-V(s)=-Z(s)=-W(s) \cdot U(s) \\
=-W(s) \cdot \frac{E}{s} \operatorname{th}\left(\frac{T s}{4}\right)
\end{gathered}
$$

The temporal evolution of $v(t)$ can be obtained by inversing Laplace transform $V(s)$.

$$
\begin{aligned}
x(t)= & \frac{1}{2 \pi j} \oint V(s) e^{s t} d s= \\
& \sum_{\text {all poles of } V(s)} \operatorname{Res}\left[V(s) . e^{s t}\right]
\end{aligned}
$$

Where $\operatorname{Res}(V(s))$ is the residue of the Laplace function $V(s)$. Since the signal is a square wave, which is periodic and symmetric, the switching instants happen at any half period $T / 2$. The switching condition at the instant $t_{i}$ according to the residue theorem is given by the closed loop condition which can be written as follows:

$v\left(t_{i}=\frac{T}{2}\right)=\oint W(s) \cdot \frac{E}{s} \cdot t h\left(\frac{T s}{4}\right) \cdot e^{s t} d s=h$

The relationship (7) gives the equation that links the period of the stable self-oscillation provided we define the transfer function $W(s)$ of the linear system under consideration.

\subsection{First order filter}

If we consider $W(s)$ as a transfer function of a first order filter of the form:

$$
W(s)=\frac{K}{\tau . s+1}=\frac{K \cdot s_{0}}{s+s_{0}}
$$

Replacing $W(s)$ from (8) into (7) and then inverting Laplace transform, we obtain: 


$$
v\left(t_{i}=\frac{T}{2}\right)=E \oint \frac{K \cdot s_{0}}{\left(s+s_{0}\right)} \cdot \frac{1}{s} \cdot t h\left(\frac{T s}{4}\right) \cdot e^{s t} d s=h
$$

As $W(s)$ has only one pole $s_{0}$, therefore by integrating (9) according to (6); we can determine the frequency of the self-oscillation $\omega=\frac{2 \pi}{T}$ w.r.t. the on-off element $(h, E)$ and the characteristic of the linear filter $\left(K, s_{0}\right)$. From (9), the frequency of self-oscillation $(w=1 / T)$ can we written in the form:

$$
T=\frac{2 \pi}{\omega}=4 \cdot \tau \cdot \arg \cdot \operatorname{th}\left(\frac{h}{K \cdot E}\right)
$$

If we consider that:

$$
\frac{h}{K \cdot E} \ll 1
$$

which is very often the case in most practical applications, then the following approximation can be used $\operatorname{th}(x) \cong x$, so, the period of self-oscillation can be written as:

$$
T=\frac{2 \pi}{\omega}=4 \cdot \tau \cdot\left(\frac{h}{K \cdot E}\right)
$$

or the frequency of self-oscillation is:

$$
\omega \cong \frac{\pi}{T} \cdot\left(\frac{K \cdot E}{\tau \cdot h}\right)
$$

We can see that this frequency is proportional to the external value of the relay, to $K$, but inversely proportional to the time response of the linear system, and to the width of hysteresis.

\subsection{Second order filter}

If we consider $W(s)$ as a transfer function of a second order filter such as:

$$
W(s)=\frac{K}{s^{2}+b \cdot s+c}=\frac{K}{s^{2}+2 \xi \cdot \omega_{n} s+\omega_{n}^{2}}
$$

Where $\left(\xi, \omega_{n}\right)$ are respectively the damping factor and the natural frequency of the system.

$W(s)$ has two poles $\left(s_{1}, s_{2}\right)$. One can write it in the equivalent form which is more convenient for applying the expression (6):

$$
W(s)=W_{1}(s)+W_{2}(s)=\frac{\omega_{n}^{2}}{s_{1}-s_{2}} \cdot\left[\frac{1}{s-s_{1}}-\frac{1}{s-s_{2}}\right]
$$

Then, applying (7), we have at the instants of commutation via the expression $[3,15]$ :

$$
\begin{gathered}
Y\left(t_{i}\right)=E \oint W(s) \cdot \frac{1}{s} \cdot \operatorname{th}\left(\frac{T s}{4}\right) \cdot e^{s t} d s \\
=E K \oint \frac{W_{1}(s)}{s} \cdot \operatorname{th}\left(\frac{T s}{4}\right) \cdot e^{s t} d s+ \\
E K \oint \frac{W_{2}(s)}{s} \cdot \operatorname{th}\left(\frac{T s}{4}\right) \cdot e^{s t} d s=h
\end{gathered}
$$

From this expression, we can extract the period $T$ of the self-oscillation, which can be expressed in the following form:

$$
\frac{1}{s_{1}} \cdot \operatorname{th}\left(\frac{T s_{1}}{4}\right)-\frac{1}{s_{2}} \cdot \operatorname{th}\left(\frac{T s_{2}}{4}\right)=\frac{h}{E \omega_{n}^{2}} \cdot\left(s_{1}-s_{2}\right)
$$

As for the first order systems, we notice that the period of self-oscillation depends on the parameters of the nonlinear element $(E, h)$ as well as on the parameters of the second order filter $\left(K, s_{1}, s_{2}\right)$. Since the denominator of the transfer function is of a second order, therefore there are three type of solutions according to the nature of the roots $\left(s_{1}, s_{2}\right)$. So, equation (17) can be rewritten for the three different cases which have been determined in papers [15]. We also notice that even in this case where the linear system is relatively simple (of the second order), the expression (17) is a transcendental equation which is not obvious to solve. In fact, the complexity to solve this problem increases with the increasing order of the linear systems since we have to add more terms according to the number of poles.

The three types of expressions depend on the $\xi$ value w.r.t the unity in the characteristic equation $[3,9,15]$.

and its:

$$
s^{2}+2 \xi . \omega_{n} s+\omega_{n}^{2}
$$

$$
\Delta=\left(2 \xi \omega_{n}\right)^{2}-4 \omega_{n}^{2}
$$

\section{a) the roots are real.}

In this case, $\Delta>0$, and $\xi>1 \Rightarrow$ the roots are real and are given as follows:

$$
s_{1,2}=-\xi \omega_{n} \pm \omega_{n} \sqrt{\xi^{2}-1}
$$

Then by subsisting $\left(s_{1}, s_{2}\right)$ in (17), it becomes:

$$
\frac{t h \frac{T}{4}\left(-\xi \omega_{n}+\omega_{n} \sqrt{\xi^{2}-1}\right)}{-\xi \omega_{n}+\omega_{n} \sqrt{\xi^{2}-1}}-\frac{t h \frac{T}{4}\left(-\xi \omega_{n}-\omega_{n} \sqrt{\xi^{2}-1}\right)}{-\xi \omega_{n}-\omega_{n} \sqrt{\xi^{2}-1}}=
$$

\section{b) the roots are equal}

In this case, $\Delta=0$, and $\xi=1 \Rightarrow$ the equation has only one double root:

$$
s_{1}=s_{2}=-\omega_{n}
$$

Then by subsisting $\left(s_{1}, s_{2}\right)$ in (17), it becomes:

$$
\frac{-\omega_{n} T+4 \operatorname{sh} \frac{\omega_{n} T}{2}}{\omega_{n}\left(1+\operatorname{ch} \frac{\omega_{n} T}{2}\right)}=\frac{h}{E \omega_{n}^{2}}
$$

c) the roots are complex

for $\Delta<0$, and $\xi<1 \Rightarrow$ the equation has two imaginary roots:

$$
s_{1,2}=-\xi \omega_{n} \pm j \omega_{n} \sqrt{\xi^{2}-1}
$$

Then by subsisting $\left(s_{1}, s_{2}\right)$ in (17), it becomes: 


$$
\frac{-\xi \sin \left(\frac{T}{2} \omega_{n} \sqrt{1-\xi^{2}}\right)+\sqrt{1-\xi^{2}} \operatorname{sh}\left(\frac{T}{2} \xi \omega_{n}\right)}{\operatorname{ch}\left(\frac{T}{2} \xi \omega_{n}\right)+\cos \left(\frac{T}{2} \omega_{n} \sqrt{1-\xi^{2}}\right)}=\frac{h}{E}\left(1+2 \xi^{2}\right) \sqrt{1-\xi^{2}}
$$

The obtained equations in the three cases are strongly nonlinear, transcendental and are difficult to solve analytically. They only can be solved by numerical techniques. The third case is particularly hard to solve because of the presence of periodic functions such as sinusoidal functions.

\section{DESCRIBING FUNCTION APPROACH}

The idea behind the use of the Describing function (DF) consists of extending the study of linear systems to nonlinear ones by means of harmonic analysis. The main purpose is to determine an equivalent transfer function for the nonlinear system $\operatorname{Gn}(a)$. The DF is based on the assumption that approximates the output signal only by its fundamental sinusoid neglecting all the other harmonic components. The DF of a nonlinearity is defined as the ratio of the fundamental of the output to the amplitude of the sinusoidal input [17]. If we consider the symmetrical relay with hysteresis defined by expressions (1), then if we impose a sinusoidal oscillation at the input of amplitude a with the angular frequency $\omega$ :

$$
e(t)=a \cdot \sin \omega t
$$

by limiting the output of the relay to its first harmonic only (fundamental sinusoid), the DF of the relay $G_{N}(a)$ can be considered as the complex gain which depends on the harmonic oscillation. It is possible to consider it like a common transfer function, as for linear systems, which can be expressed as follows $[2,9]$ :

$$
G_{N}(a)=\left\{\begin{array}{cc}
\left.\frac{4 E}{\pi a} \sqrt{1-\left(\frac{h}{a}\right)^{2}}-j \frac{h}{a}\right] & \text { for } 0 \leq h<a \\
0 & \text { for } 0 \leq a<h
\end{array}\right.
$$

Because of neglecting the harmonics, thus, the DF approach gives more accurate results if the behavior of the linear systems is close to a behavior of a low-pass filter [2, 7]. To determine the oscillation of the system, we use the framework of linear systems. The oscillation rises only when the system is marginally stable. The necessary condition for the system to be marginally stable is $[3,4,7]$.

$$
G o(j \omega)=N(j \omega) \cdot G(j \omega)=-1
$$

This relationship will be applied to first and second order linear systems for determining the self-oscillations occurring in the corresponding feedback control systems.

\subsection{DF for First Order Linear systems}

In case of linear systems of the first order as given in (8), the condition of stability limit (28) can be rewritten by separating the non-linear and the linear part as follows:

$$
-\frac{1}{N(a, \omega)}=W(\omega)
$$

from (26), we have:

$$
W(\omega)=-\frac{1}{N(a, \omega)}=-\frac{\pi}{4 \cdot E} \cdot\left(\sqrt{a^{2}-h^{2}}+j h\right)
$$

and rewriting (8) in function of the frequency of the selfoscillation as follows:

$$
W(\omega)=\frac{K s_{0}}{s_{0}^{2}+\omega^{2}}+j \frac{-K s_{0} \cdot \omega}{s_{0}^{2}+\omega^{2}}
$$

By equaling the imaginary parts (30) to (31) as indicated in (29), we can obtain the equality that contains the frequency of the self-oscillation which is:

$$
\frac{\pi h}{4 . E}=\frac{k s_{0} \cdot \omega}{s_{0}^{2}+\omega^{2}}
$$

This last relation (31) can be written in a polynomial form which links the frequency of the self-oscillation w.r.t to the parameters of the feedback control system:

$$
\omega^{2}-\frac{4 K E s_{0}}{\pi h} \cdot \omega+s_{0}^{2}=0
$$

Solving this equation provides two solutions according to delta:

$$
\Delta=4 . s_{0}^{2}\left[\left(\frac{2 K E}{\pi \cdot h}\right)^{2}-1\right]=4 . s_{0}^{2}\left(\frac{2 K E}{\pi \cdot h}\right)^{2}\left[1-\left(\frac{\pi \cdot h}{2 K E}\right)^{2}\right]
$$

We obtain two solutions and the realistic one has to correspond to a stable self-oscillation:

$$
\omega_{1,2}=\frac{2 K \cdot E \cdot s_{0}}{\pi \cdot h} \pm \frac{\sqrt{\Delta}}{2}
$$

\subsection{DF for Second order linear systems}

In case of second order linear systems, we consider a transfer function in the general form given in (14). To estimate the frequency of the self-oscillation that is generated by the relay feedback control, we use again the relationship (29). As we have proceeded for the first order linear system; we compute the imaginary part of $W(\omega)$ which is:

$$
\operatorname{Im}(W(\omega))=\frac{-b K \omega}{\left(c-\omega^{2}\right)^{2}+\mathrm{b}^{2} \omega^{2}}
$$

by comparing the two imaginary parts, we establish the equality:

$$
-\frac{1}{N(a, \omega)}=W(\omega)=-\frac{\pi h}{4 M}=\frac{-a K \omega}{\left(b-\omega^{2}\right)^{2}+\mathrm{a}^{2} \omega^{2}}
$$

from which, we can get the polynomial equation that provides the self-oscillation $\omega$ with respect to the parameters of the feedback control system:

$$
\omega^{4}+\left(a^{2}-2 b\right) \omega^{2}-\frac{4 \cdot a K \cdot M}{\pi h} \cdot \omega+b^{2}=0
$$

in the case of second order linear systems, the solutions cannot be obtained easily but can be instead computed by means of numerical techniques. It can also be computed and visualized from graphics provided by simulation or experimental systems. The previous equations give four 
solutions. Nyquist's diagram can determine the stable self-oscillation.

\section{SIMULATION AND EXAMPLES}

\subsection{First order filter}

For the simulation of the feedback control system with a first order filter, we have used the following feedback control model (Figure 3). In this Figure, the relay characteristics are chosen: $h=1, E=12$. Given the relay characteristics and fixing $K=1$, the only parameter that influences the self-oscillation is the time constant parameter $\tau$. To determine the frequency of the stable selfoscillations, we modify the value of so and we compute the roots of the polynomial equation (32).

Then, we use Nyquist criterion to select the stable frequency (Figure 4.). Figure 5 presents a graphical representation, obtained by simulation, of the output $u(t)$ of the relay and the output $v(t)$ of the linear system. The theoretical value of the frequency of the self-oscillation can be calculated from the Tsypkin's expressions (10) and (12); and measured from the graphics on Figure 5.

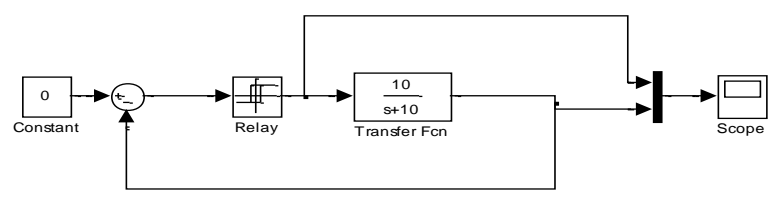

Figure 3. Scheme with a first order system with Simulink

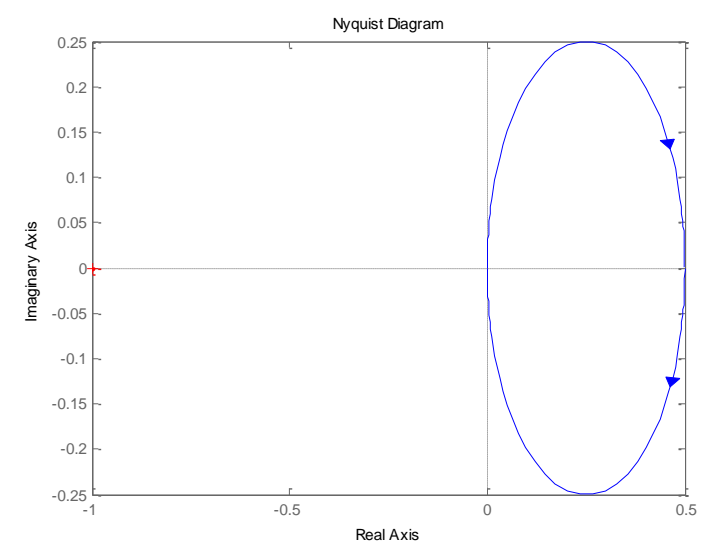

Figure 4. Diagram of Nyquist of linear system

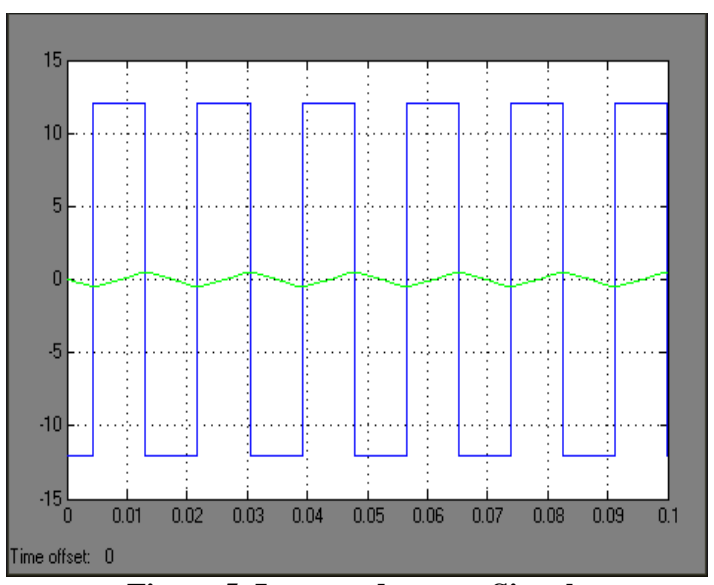

Figure 5. Input and output Signals

In Table 1, we present some values of the frequency of the self-oscillations obtained by Tsypkin's approach $\left(\omega_{\text {Tsypkin }}\right)$ and by DF approach $\left(\omega_{\mathrm{DF}}\right)$ with respect to the cutting frequency of the linear system $s_{0}$. we present also the relative error which is defined as:

$$
e=\frac{\omega_{\text {Tsypkin }}-\omega_{\mathrm{DF}}}{\omega_{\text {Tsypkin }}}
$$

Table 1. Margins frequency estimation and relative errors for a first order system

\begin{tabular}{|l|l|l|l|}
\hline \multicolumn{1}{|c|}{$s_{0}$} & \multicolumn{1}{|c|}{$\omega_{\mathrm{DF}}$} & $\omega_{\text {Tsypkin }}$ & $\begin{array}{c}\text { Relative error } \\
e\end{array}$ \\
\hline $\begin{array}{l}0.01 * 2 * \pi= \\
0.0628\end{array}$ & 0.1522 & 0.1884 & 0.19214 \\
\hline $0.1 * 2 * \pi=0.628$ & 1.5221 & 1.8840 & 0.19209 \\
\hline $2 * \pi=6.283$ & 15.2209 & 18.840 & 0.19209 \\
\hline $10 * 2 * \pi=62.83$ & 152.2093 & 188.40 & 0.19209 \\
\hline $100 * 2 * \pi=628.3$ & 1522.102 & 1888.4 & 0.19397 \\
\hline
\end{tabular}

Since $\omega_{\text {Tsypkin }}$ is considered as an accurate solution, our results show that the relative error on $\omega_{\mathrm{DF}}$ is almost constant and independent from the parameters of the feedback control systems. the numerical results show that this error is bounded by the number $19,4 \%$.

Determination of the error between the two approaches To have a rough estimation of the error between the two approaches (Tsypkin and DF), we use the approximation (11). Within this approximation, the frequency of selfoscillations has been already determined by Tsypkin's approach in relation (13).

By applying the same approximation to the expression $\Delta$ from (34), the square root of $\Delta$ is:

$$
\sqrt{\Delta}=2 \cdot s_{0} \cdot\left(\frac{2 K E}{\pi \cdot h}\right)\left[1-\left(\frac{\pi \cdot h}{2 K E}\right)^{2}\right]^{0.5}
$$

by using the following Taylor's approximation to the first order:

$$
(1+\varepsilon)^{0.5} \cong 1-0.5 \varepsilon
$$

and applying it to the expression: 


$$
\left[1-\left(\frac{\pi \cdot h}{2 K E}\right)^{2}\right]^{0.5}=1-0.5\left(\frac{\pi \cdot h}{2 K E}\right)^{2}
$$

The expression of the square root (40), becomes:

$$
\sqrt{\Delta}=2 \cdot s_{0} \cdot\left(\frac{2 K E}{\pi \cdot h}\right)\left[1-0.5\left(\frac{\pi \cdot h}{2 K E}\right)^{2}\right]
$$

By replacing this last approximation in expression (39), we can estimate the frequency of the self-oscillation given by DF approach from the expression (34) which becomes:

$$
\omega_{D F} \cong \frac{4}{\pi}\left(\frac{s_{0} K E}{h}\right)
$$

If we consider the approximation of the frequency of the self-oscillation given by Tsypkin approach in (13) which is:

$$
\omega_{T S} \cong \frac{\pi}{2}\left(\frac{K E}{h}\right)
$$

Then, we can estimate the relative error obtained under these approximations which is:

$$
e=\frac{\omega_{T S}-\omega_{D F}}{\omega_{T S}} \cong 1-\frac{8}{\pi^{2}} \cong 0.194
$$

This result is in close to the one reported by calculations in Table.1. This means that for linear systems of first order, the relative error is constant and bounded by (19.4 $\%)$. It does not vary with the variation of the other system parameters. However, this result holds as far as the approximation $\left(\frac{h}{K E}\right) \leq 1$ is respected. As a consequence, from (10), this approximation leads to the following one too: $\frac{T}{\tau}=\frac{s_{0}}{\omega} \ll 1$. This means that the period of the selfoscillation is always largely less than the cutting frequency, which means again that the generated frequency is always much higher that the cutting frequency. In conclusion, under this approximation, the filter is always a low-pass one which justifies with some error, the use of the DF method.

However, if the approximation $\left(\frac{h}{K E}\right) \leq 1$ or equivalently $\frac{T}{\tau}=\frac{s_{0}}{\omega} \ll 1$ is not respected, this means that the frequency of the self-oscillation becomes closer to or less than the cutting frequency, and then the filtering becomes weak. This means that the distortion becomes non negligible and therefore the assumption concerning the DF becomes not accurate and less or even not applicable.

\subsection{Second order filter}

Similarly, as with the first order linear systems, simulation of the feedback control system including second order linear systems was performed by means of Simulink (Figure 6). The relay characteristics are: $h=1, E=12$. We proceed by modifying some values of the two parameters that characterizes the second order filter $(b, c)$ or equivalently $\left(\xi, \omega_{n}\right)$ and then we compute the corresponding frequency of self-oscillations for stable vibrations (eq 14). Let's establish the relations between the parameters $(b, c)$ and $\left(\xi, \omega_{n}\right)$ which are:

$$
b=2 \xi \omega_{n} \quad c=\omega_{n}^{2} \quad \xi=\frac{1}{2} \frac{b}{\sqrt{c}}
$$

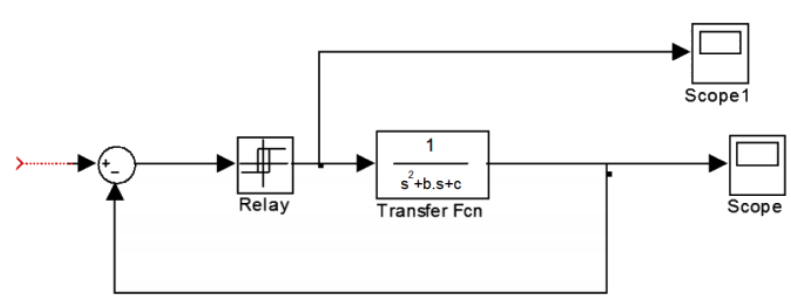

Figure 6. Scheme of the 2nd order system with Simulink For both approaches, the theoretical equations that determine the frequency of self-oscillations are strongly nonlinear. So, solving the corresponding equations can only be done via numerical computations. Concerning the $\mathrm{DF}$ approach, it is relatively easy to solve the equation for the first order system, but it is hard to solve the polynomial equation (11) for the second order system.

In the same way, with Tsypkin's approach, it is easy to find the solution for the first order system but it is difficult to solve the equations for the second order filter. To determine $\omega_{\mathrm{DF}}$, for the second order system, there are many available solvers for polynomial equations. It is more difficult to solve the nonlinear equations derived from Tsypkin's approach for the second order system. Numerical techniques and simulation have been used for determining $\omega_{\text {Tsypkin }}$. experiments have been also used as well as simulation for visualizing the behavior of the system to check the numerical values as far as it is possible.

Table 2 presents the frequency of the self-oscillations as estimated by Tsypkin's approach and by DF approach with respect to the parameters $(b, c)$ or $\left(\xi, \omega_{n}\right)$. The estimation of the relative error is also reported in Table 2. In fact, the determination of the frequency of selfoscillations have been carried out in the case of $\xi>1$. Even for this case, the determination of some frequencies was not possible to determine by methods: solvers, simulation and experiments (see the last line of Table 2). The case where $\xi<1$, the problem is harder. We have also selected the values of $\left(\xi, \omega_{n}\right)$, so, that the poles of the transfer function $\left(s_{1}, s_{2}\right)$ are negative which ensures the stability of the feedback control system. The values of $\omega_{\text {Tsypkin }}$ have been determined by means of the simulation. The values of $\omega_{\mathrm{DF}}$ have been determined by numerical computation.

Table 2. Frequency estimations for a second order linear system 
According to Table 2, the estimation of $\omega_{\mathrm{DF}}$ is much better for second order linear systems than for the first order linear systems. Even under the approximation used in (10), the relative errors vary w.r.t the coefficients $\left(\xi, \omega_{n}\right)$ of the linear systems. However, the errors are less important compared to the first order linear systems. This is certainly due to the fact that system of the second order reacts as two serial first order systems and therefore the filtering is improved.

\section{EXPERIMENTS}

An experimental setup has been built to experiments the behavior of feedback control systems. A validation of the theoretical approach of Tsypkin has been performed successfully. Some applications studied such as converters based on PWM and optimal tracking systems [19]. The relay is constituted by a Schmitt trigger based on transistors. Figure 7 presents a picture of our experimental setup visualizing simultaneously on a PC and on an oscilloscope the input and output of the relay. Another picture shows on the oscilloscope, the input and the output signals of the electronic relay.

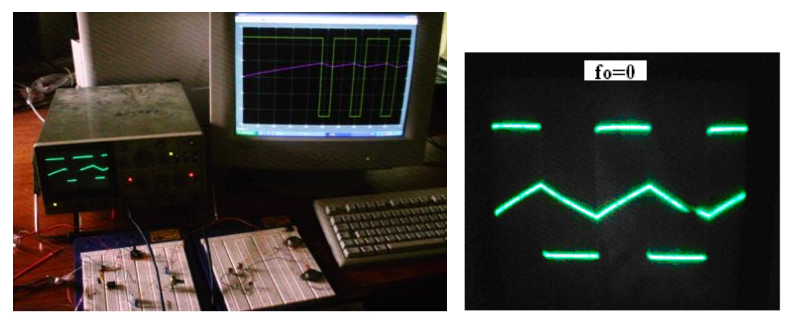

Figure 7. Experimental setup.

\section{CONCLUSIONS}

We have presented analytical expressions for determining the frequency of self-oscillations based on Tsypkin's approach in case of linear systems. The expressions reveal to be difficult to solve for linear systems over than the first order system because they combine transcendental functions. In the same context, we have presented the analytical expressions for determining the frequency of self-oscillations based on the Describing Function approach. The solutions are approximations and have been written in a polynomial form and have been obtained via polynomial equation solvers. Nyquist diagram is used for selecting the root that corresponds to the stable frequency.

We have analyzed and tested these two methods for linear systems of a first and second order. For the second order systems, we can experience difficulties to find solutions for some parameters of the feedback control systems. Even simulations can fail because of the complexity of calculation. even, experimental systems are limited for a range of high frequencies because of the time response of the switching components used. Of course, for higher order linear systems, the complexity of the mathematical expressions grows up.

\begin{tabular}{|c|c|c|c|c|}
\hline $\begin{array}{c}\text { Coefficients } \\
(b, c)\end{array}$ & $\begin{array}{c}\text { Coefficients } \\
\left(\xi, \omega_{n}\right)\end{array}$ & $\omega_{\text {Tsypkin }}$ & $\omega_{\mathrm{DF}}$ & $\begin{array}{c}\text { Relative } \\
\text { error: } \mathrm{e}\end{array}$ \\
\hline $\begin{array}{c}b=4, \\
c=1\end{array}$ & $\begin{array}{c}\xi=2, \\
\omega_{n}=1\end{array}$ & 2.80 & 2.79 & 0.0036 \\
\hline $\begin{array}{c}b=1, \\
c=0.01\end{array}$ & $\begin{array}{c}\xi=5, \\
\omega_{n}=1\end{array}$ & 2.33 & 2.347 & -0.0073 \\
\hline $\begin{array}{c}b=1, \\
c=0.1\end{array}$ & $\begin{array}{c}\xi=1.58, \\
\omega_{n}=0.386\end{array}$ & 2.33 & 2.37 & -0.017 \\
\hline $\begin{array}{c}\xi=10, \\
c=1\end{array}$ & $\begin{array}{c}\xi=5, \\
\omega_{n}=1\end{array}$ & 1.69 & 1.51 & 0.106 \\
\hline $\begin{array}{c}b=10, \\
c=0.1\end{array}$ & $\begin{array}{c}\xi=15, \\
\omega_{n}=0.316\end{array}$ & 1.65 & 1.497 & -0.092 \\
\hline $\begin{array}{c}b=100, \\
c=10\end{array}$ & $\begin{array}{c}\xi=15.80, \\
\omega_{n}=3.16\end{array}$ & $\begin{array}{c}\text { No } \\
\text { oscillation }\end{array}$ & $\begin{array}{c}\text { No } \\
\text { oscillation }\end{array}$ & \multicolumn{1}{|l}{} \\
\hline
\end{tabular}

The comparison of these two approaches has been performed for nonlinear systems of first and second orders leading to understand better the behavior of such systems and the limit of the tools to be used for determining and estimating the frequencies of self-oscillations. The experimental feedback control system based on on-off electronic circuits with hysteresis was build up and was successfully used to visualize the behavior of the feedback control systems. It is enabling to envision many interesting applications.

\section{REFERENCES}

[1] Atherton D. P., (1996). Early developments in nonlinear control. in IEEE Control Systems Magazine, vol. 16, no. 3, pp. 34-43.

[2] Holmberg U., (1991). Relay Feedback of Simple Systems. Department of Automatic Control, Lund Institute of Technology (LTH), (PhD Thesis TFRT1034).

[3] Tsypkin Ya-Z, (1984). Relay Control Systems, Cambridge University Press, Cambridge, UK.

[4] Hamel B, (1956). A mathematical study of on-off controlled higher order systems. in Proceedings of the Symposium on Nonlinear Circuit Analysis, Polytechnic Institute of Brooklyn, New York, volume 6, pp. 225-232.

[5] Astrom, K.J. and Hagglund, T., (1984). Automatic tuning of simple reg-ulators with specifications on phase and amplitude margins. Automatica, Vol. 20, No. 5, pp. 645-651.

[6] Migkovic, N. and Vukic, Z. and Barisic, M., (2007). Transfer function identification by using selfoscillations. Mediterranean Conference on Control \& Automation, Athens, pp. 1-6.

[7] Tianshi Wang., (2017). Analyzing Oscillators using Describing Functions. arXiv:1710.02000.

[8] Michael Ruderman., (2019). Relay Feedback Systems - Established Approaches and New Perspectives for Application. IEEJ Journal of Industry Applications, Vol. 8, No. 2, pp 271-278,

https://doi.org/10.1541/ieejiia.8.271

[9] Boiko, I., (2004). Analysis of modes of oscillations in a relay feedback system. Proceedings of the 2004 American Control Conference, Boston, MA, USA, pp. 1253-1258 vol.2. 
[10] Fielding, C., and Flux, P., (2003). Non-linearities in flight control systems. The Aeronautical Journal (1968), Vol. 107, No. 1077, pp. 673-686. doi: $10.1017 / \mathrm{S} 0001924000013543$

[11] Yoon YE, and Johnson EN., (2017). Analysis of simple relay feedback adaptive control. In AIAA Guidance, Navigation, and Control Conference. American Institute of Aeronautics and Astronautics Inc, AIAA. 2017. (AIAA Guidance, Navigation, and Control Conference, 2017).

[12] Dipesh Shah et al., (2018). State Feedback Control for Cruise Missile System; International Conference on New Trends in Engineering and Technology (IEEE Madras Section).

[13] Miskovic. N., et al., (2009). IFAC Proceedings, Vol. 42 , No. 18 , pp. $115-120$

https://doi.org/10.3182/20090916-3-BR-3001.0025

[14] R. Gupta and A. Ghosh. (2006) Frequency-domain characterization of sliding mode control of an inverter used in DSTATCOM application. In IEEE Transactions on Circuits and Systems I: Regular Papers, vol. 53, no. 3, pp. 662-676.

[15] Zaatri A., and Belhour S., (2014). Analysis and Design of a PWM Chopper -On/Off Control via a second order filter. World Journal of Engineering. 11(2),181-186, 2014.

[16] Eckman, D.P., (1954). Phase-plane analysis. A general method of solution for two-position process control. Trans. ASME, 76, pp. 109-116

[17] Derek Atherton., (2011) An Introduction to Nonlinearity in Control Systems. bookboon.com. ISBN 978-87-7681-790-9

[18] Panda, Rames and Yu, Cheng-Ching., (2003). Analytical Expressions for Relay Feedback Responses. Journal of Process Control. 13. 489-501. 10.1016/S0959-1524(02)00119-1.

[19] Zaatri A., and Belhour S., (2010). Etude et réalisation d'un hacheur PWM. Revue des Energies Renouvelables Vol.13 No 1. pp 187-198 